\title{
Impacto social y económico del Corredor Vial Cañete - Perú
}

\author{
Social and economic impacts of Cañete Road Corridor case - Peru \\ Impacto social e econômico do Corredor Rodoviário de Cañete - Peru
}

\section{ARTÍCULO GENERAL}

\author{
Ricardo Walter Soto Sulca \\ https://orcid.org/0000-0002-9936-0609 \\ rsoto@uncp.edu.pe \\ Facultad de Trabajo Social, Universidad Nacional \\ del Centro del Perú (UNCP), Huancayo-Perú.
}

\author{
Julissa Karent Muñoz Rojas \\ https://orcid.org/0000-0003-3320-5064 \\ jmunioz@,uncp.edu.pe \\ Facultad de Trabajo Social, Universidad \\ Nacional del Centro del Perú (UNCP), \\ Huancayo-Perú.
}

\author{
Yulia Nadia Cáceres Quispe \\ https://orcid.org/0000-0001-5968-9912 \\ yuliacq@uncp.edu.pe \\ Facultad de Trabajo Social, Universidad \\ Nacional del Centro del Perú (UNCP), \\ Huancayo-Perú.
}

Recibido 07 de Junio 2021 | Arbitrado y aceptado 25 de Agosto 2021 | Publicado en 04 Diciembre 2021

\section{RESUMEN}

Uno de los ejes centrales para el desarrollo económico y social de cualquier país es la construcción de carreteras, dado que estas vías de comunicación terrestre además de contribuir a la planificación y al ordenamiento territorial impulsa el crecimiento demográfico, económico y social de sus comunidades. La presente investigación identifica los impactos sociales y económicos, generados por la construcción del Corredor Vial Cañete en sus ámbitos territoriales, para realizar la investigación se aplicó la técnica de la encuesta, entrevista y la revisión bibliográfica, haciendo uso del método análisis síntesis. Los resultados mostraron que el impacto social que ha producido en los pobladores del Corredor vial Cañete ha sido: el mayor acceso a la educación, salud y a mejoramiento de servicios básicos y el impacto económico ha sido: mayor acceso a los mercados para los cultivos y productos locales, el acceso a nuevas oportunidades de empleo, desarrollo de nuevas actividades económicas como el turismo y el fortalecimiento de la economía.

Palabras Clave: Impacto social, impacto económico, Corredor Vial Cañete, educación, salud, servicios básicos, acceso, mercados, empleo.

\section{ABSTRACT}

One of the central axes for the economic and social development of any country is the construction of highways, since these roads of land communication, in addition to contributing to planning and land use, promote the demographic, economic and social growth of their communities. This research identifies the social and economic impacts, generated by the construction of the Cañete Road Corridor in its territorial areas, to carry out the research the survey, interview and bibliographic review technique was applied, making use of the synthesis analysis method. The results showed that the social impact that it has produced on the inhabitants of the Cañete road corridor has been: greater access to education, health and improvement of basic services and the economic impact has been: greater access to markets for crops and products premises, access to new employment opportunities, development of new economic activities such as tourism and the strengthening of the economy.

Key Words: Social impact, economic impact, Cañete Road Corridor, education, health, basic services, access, markets, employment.

\section{RESUMO}

Um dos eixos centrais para o desenvolvimento econômico e social de qualquer país é a construção de rodovias, uma vez que essas vias de comunicação terrestre, além de contribuir para o planejamento e uso do solo, promovem o crescimento demográfico, econômico e social de suas comunidades. Esta pesquisa identifica os impactos sociais e econômicos, gerados pela construção do Corredor Viário do Cañete em suas áreas territoriais, para a realização da pesquisa foi aplicada a técnica de levantamento, entrevista e revisão bibliográfica, utilizando o método de análise-síntese. Os resultados mostraram que o impacto social que produziu sobre os habitantes do corredor rodoviário de Cañete foi: maior acesso à educação, saúde e melhoria dos serviços básicos e o impacto econômico foi: maior acesso a mercados para instalações de lavouras e produtos, acesso a novas oportunidades de emprego, desenvolvimento de novas atividades econômicas como o turismo e o fortalecimento da economia.

Palavras-chave: Impacto social, impacto econômico, Corredor Rodoviário de Cañete, educação, saúde, serviços básicos, acesso, mercados, emprego. 
Introducción

El trabajo y esfuerzo que dedica el sector de transportes y telecomunicaciones en función al reto propuesto de vencer las brechas existentes en infraestructura y servicios que involucran el transporte; supone desde ya, enfrentar estos desafíos con responsabilidad para llevar al Perú a una ventaja competitiva y verse a sí mismos como un país en desarrollo. "Estos desafíos y el actual contexto, nos han llevado a reformular nuestras prioridades y estrategias, tomando en cuenta las brechas pendientes de cerrar, las necesidades y carencia de nuestra población objetivo y enmarcada en la Política General de Gobierno y el Plan Estratégico de Desarrollo Nacional" (PESEM: Sector de transporte y comunicaciones 20182023).

Según el reporte de competitividad del WEF (Foro Económico Mundial) del período 2018, “el Perú se encuentra en el puesto 63 del ranking de 140 países. En la Red Vial Departamental sólo un poco más del $20 \%$ de su extensión total está en buen estado". Asimismo, el Índice de Desarrollo de las TIC (IDT) de la UIT 2017, señala que "el Perú tiene un índice de 4.85, muy inferior al promedio de los países de la Alianza del Pacífíco (5.7) y de otras regiones avanzadas". El deficiente desarrollo de las telecomunicaciones se produce por las siguientes razones: i) escaza cobertura de los servicios (solo $30 \%$ de las ciudades con más de 100 residentes tienen el servicio de internet), ii) bajo uso (49\% de la población hacen uso del servicio de internet) y iii) deficiente asequibilidad ( $21 \%$ de hogares no son usuarios del servicio de internet fijo ya que sus tarifas son altas).

Los gobiernos regionales mantienen escasos recursos para su mantenimiento, a consecuencia de ello se observa que la mayor parte de esta posee un estado entre regular y mala conservación, presentando un mantenimiento monótono e insuficiente. Además, más de 50,000 Km. inventariados vienen a ser trochas con condiciones técnicas bajas y no responden a los estándares técnicos necesarios para implementar programas de mantenimiento adecuados. Las carreteras son un importante recurso para el desarrollo socioeconómico del país, la globalización y las necesidades sociales ponen en evidencia la necesidad de brindar una mejor calidad y eficiencia del sistema de comunicación a través del

Ministerio de transportes y comunicaciones con el objetivo de lograr un impacto positivo en el desarrollo local, regional y nacional. 
Según SUTRAN, "los niveles de cumplimiento de la normativa en el transporte de personas y en el de mercancías fue $67.74 \%$ en el año 2016", esta tasa indica el grado de adecuación de las empresas de transporte terrestre de carga y pasajeros a las exigencias de la normativa nacional. "A inicio del año 2000 comienzan las construcciones de las vías de comunicación en la mayoría de los distritos y pueblo de la sierra central (Junín, Huancavelica y Ayacucho), financiados por el Ministerio de Transporte o las municipalidades distritales. "Para alcanzar las reformas deseadas a nivel nacional, el Ministerio de Economía y Finanzas (MEF) del Perú recibe el apoyo económico y técnico del Banco Mundial”. "En los últimos años, se ha logrado la mejora de algunos aspectos en las vías de transporte al interior del país y sus conexiones con el exterior, a fin de promover la importación y exportación; asimismo, se han logrado mejoras en los sectores de educación y salud" (Meza S, 2016).

La transformación en la zona rural y en las urbanas recepcionistas de intensas migraciones, así como la transformación rápida de los patrones culturales, ha estado ligada a otros procesos como el incremento de carreteras, medios de transporte externo e interno, junto a una tardía presencia del Estado. Es decir, una modernización y dinamización de las amplias zonas rurales de la sierra central y del país, donde habitaban, fundamentalmente, las poblaciones campesinas. Sin embargo, para que exista tal movimiento, recalca Puyol R, (2012) "El desarrollo de la población ciudadana será uno de los grandes vectores del cambio social que se avecina se requiere la constitución de sistemas técnicos de canales, carreteras, a través de los cuales puede ocurrir el movimiento"

Las carreteras son un nexo o suerte de comunicación para las zonas campesinas, ya que acorta la distancia que separa a los centros poblados, distritos y las grandes ciudades del valle de Mantaro, parte de la zona de Huancavelica y Lima. Estas contribuyen en el establecimiento de lazos comunitarios puesto que la construcción de carreteras en los distritos rurales y lejanos de las ciudades, a través de las municipales distritales, municipalidades provinciales, gobiernos regionales y Ministerio de Trasporte han venido modificando la relación entre los residentes de pueblos lejanos y los migrantes que radican en la ciudad, ya que sus relaciones son cada vez más permanentes entre ellos. Esto se puede constatar en la dinámica socioeconómica de las comunidades campesinas del valle del Mantaro y en la bibliografía identificada. 
"La construcción del Corredor Vial Cañete es considerada un importante corredor para mejorar la accesibilidad a la zona centro del país, pues agrupa provincias desde Lunahuaná - Dv. Yauyos hasta la división Dv. Pampas y permite integrar dos regiones del Perú: la costa con la sierra" (desde Cañete hasta

Huancayo). (MTC 2010)

En ese sentido la construcción del Corredor Vial Cañete mejoraría el acceso a la zona centro del país y la articulación entre tres regiones ; Junín,

Huancavelica y Lima, no obstante dicho corredor se caracteriza por tener zonas geográficamente accidentados convirtiéndolos en vías de comunicación difíciles de acceder, como también posee poblados con trochas corrosibles lo que provoca un transporte riesgoso e impacta negativamente al bienestar social, dificultando el acceso a la educación y al transporte de productos agrícolas por ende al desarrollo económico. Por ello no basta con analizar estudios geográficos, también es necesario complementarlos con análisis de índole socioeconómico, que permitan observar consecuencias positivas de las inversiones públicas en infraestructura, lo cual generaría un eficiente impacto en los ingresos económicos de los hogares, la productividad agrícola, mejora de la salud y el acceso a la educación. Por ello se resalta la importancia de la construcción de dicha carretera que contribuiría con la mejora de la calidad de vida de los pobladores y al crecimiento de PBI. Por lo tanto, la presente investigación analiza el impacto económico y social que atravesaron los pobladores beneficiarios de la construcción de la carretera, considerando además que es preciso contar con información que refleje dicho impacto desde diversos enfoques.

\section{Materiales y Métodos}

Es un estudio de diseño no Experimental

Descriptiva, se realizó el análisis exhaustivo de los cambios que se dieron en la población por medio de la construcción del corredor vial en la zona de influencia. La investigación fue de corte mixto: cuantitativo y cualitativo las técnicas empleadas para la recolección de datos fueron: la encuesta, la entrevista y la revisión bibliográfica utilizándose el método de análisis síntesis. Los participantes en el estudio fueron 20 comunidades, provincias y distritos de la población al pie de la carretera del Corredor Vial Cañete, se eligieron a informantes claves a quienes se les aplicaron la encuesta conformado por: autoridades, docentes y personal de salud, y pobladores para las entrevistas. 
Resultados

Los principales lugares donde el corredor vial tiene influencia, se encuentran distribuidos en los siguientes tramos:

- $\quad$ Tramo I: San Vicente y Nueva Imperial

- $\quad$ Tramo II: Nueva imperial - Lunahuana

- $\quad$ Tramo III: Lunahuana - Zúñiga

- $\quad$ Tramo IV: Zúñiga - Tomas

- $\quad$ Tramo V: Salida de Tomas - Tinco Yauricocha

- $\quad$ Tramo VI: Tinco Yauricocha - Roncha

- $\quad$ Tramo VII: Roncha - Pucara

- $\quad$ Tramo VIII: Pucara - Pazos

- $\quad$ Tramo IX: Pazos - División Pazos

- Tramo X: División San Vicente de Cañete - Cañete

En estos tramos se encuentran tres regiones que corresponde a Lima, Junín y Huancavelica por ello se describe de manera general aspectos fundamentales para la presentación de resultados.

Impacto Social

En relación al aspecto social se dividen en diversas variables como son educación, salud y servicios básicos.

\section{Educación}

Se describe los impactos generados por el proyecto en relación a la dimensión de educación e inclusión social, teniendo como eje principal el acceso a la educación en las diversas instituciones educativas de los diferentes niveles: primario, secundario y universitario.

Actualmente la población beneficiaria que cuenta con acceso a educación asciende a un total de 24616 de población escolar ubicados en los tramos de estudio, que a continuación se detalla: 


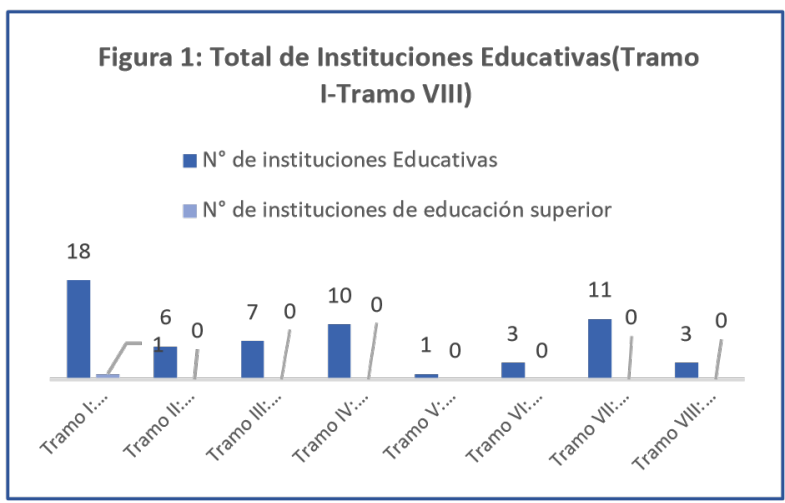

Fuente: Encuesta aplicada a pobladores por cada tramo--2018

Se muestra el número de instituciones educativas ubicadas en los diferentes tramos: Tramo I (13 instituciones educativas y 1 institución de educación superior) Tramo II (6), Tramo III (7), Tramo IV (10) y

Tramo V (1), Tramo VI (3), Tramo VII

(11) y Tramo VIII

(3).

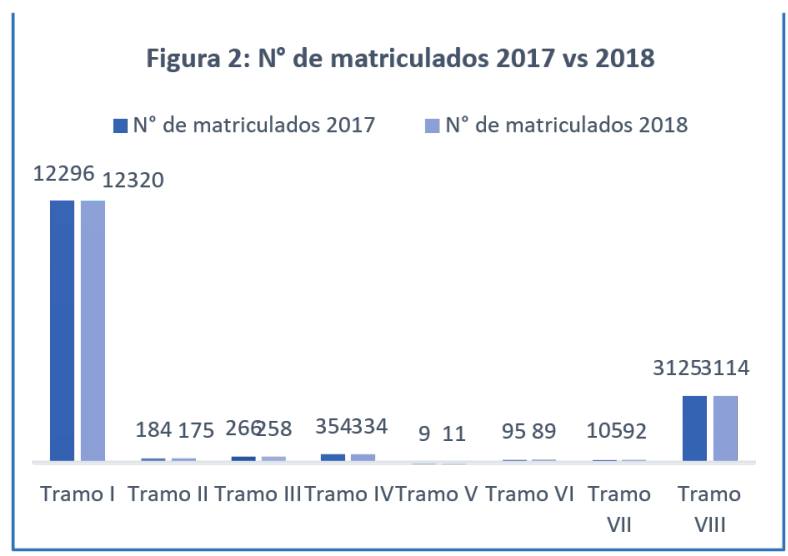

Fuente: Entrevista aplicada a docentes de las diferentes instituciones educativas ubicados del tramo I al tramo VIII. -2018

Se muestra el número de matriculados en las diferentes instituciones educativas ubicados en los tramos del I al IV en el año 2017 y 2018. En el 2018 en el Tramo I y en el Tramo $\mathrm{V}$, existe un incremento en el número de matriculados a diferencia del año 2017,

Asimismo, al preguntar a los docentes (informantes claves) acerca de si la construcción del Corredor Vial Cañete, 
mejoro el servicio educativo de su zona, Tramo III:

respondieron:

Tabla 1: Impacto de la canstrucción del Corredor

Lunahuana -

Zự̂iga

Vial

Caniete en el senvicio de educación

\begin{tabular}{|c|c|}
\hline Tramo & Informante Clave \\
\hline $\begin{array}{l}\text { Tramo I: San } \\
\text { Vicente - } \\
\text { Imperial }\end{array}$ & 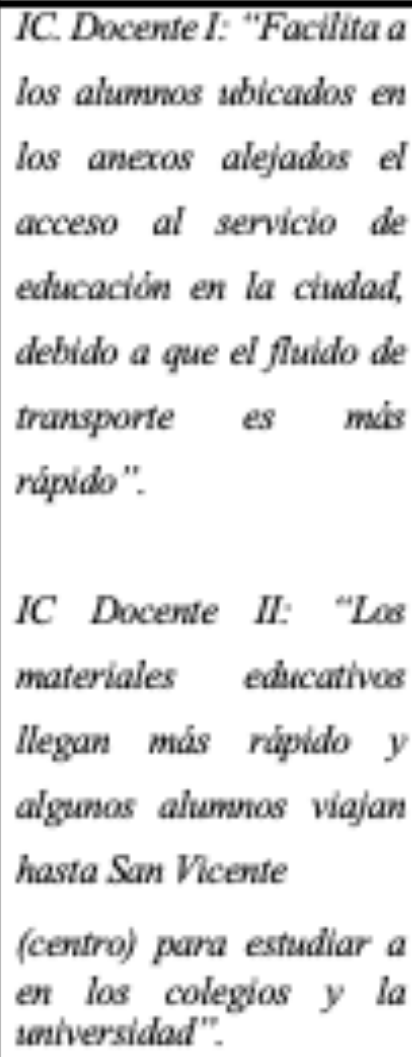 \\
\hline $\begin{array}{l}\text { Tramo II: } \\
\text { Nueva } \\
\text { Imperial - } \\
\text { Lunahuana }\end{array}$ & $\begin{array}{l}\text { IC-Docente I: "Ayndo a } \\
\text { que los estudiantes se } \\
\text { puedan transpontar } \\
\text { facilmente". } \\
\text { IC- Docente II: "Antes } \\
\text { madrugaban pura ir a } \\
\text { estudiar, iban caminando } \\
\text { ahora hay carnos". }\end{array}$ \\
\hline
\end{tabular}

IC. Docente II: "Ahara las estudiantes se trasladan en moto taxi a Pacarin a estadiar o se van a estudiar a Imperial Lamahuana".

\begin{tabular}{|c|c|}
\hline $\begin{array}{l}\text { Tramo IV: } \\
\text { Zữiga - } \\
\text { Tomas }\end{array}$ & $\begin{array}{l}\text { IC-Docente I: "A los } \\
\text { aliminos ayuda a llegar a } \\
\text { sus centros de estudios y } \\
\text { pueden ser más } \\
\text { puntuales". } \\
\text { IC- Docente II: "Si ayuda } \\
\text { a los estudiantes a que } \\
\text { puedan ìr a estudiar y } \\
\text { volver a sius casas, de } \\
\text { manera más rápida". }\end{array}$ \\
\hline $\begin{array}{l}\text { Tramo V: } \\
\text { Tomas - Tinco } \\
\text { Yauricocha }\end{array}$ & $\begin{array}{l}\text { IC-Docente } I \text { "Sl, ahora } \\
\text { la municipalidad de Alis } \\
\text { les pone la movilidad a } \\
\text { los estudiantes para ir al } \\
\text { colegio" }\end{array}$ \\
\hline $\begin{array}{l}\text { Tramo VI: } \\
\text { Tinco } \\
\text { Yauricocha - } \\
\text { Roncha. }\end{array}$ & $\begin{array}{l}\text { IC- Docente i:" Antes } \\
\text { venian caminando ahora } \\
\text { vienen con movilidad los } \\
\text { estudiantes de los otros } \\
\text { anexas". }\end{array}$ \\
\hline
\end{tabular}




\begin{tabular}{|l|l|}
\hline & $\begin{array}{l}\text { IC-Docente II: Si porque } \\
\text { de los niños pueden viajar } \\
\text { a su colegio } \\
\text { rápidamente". }\end{array}$ \\
\hline $\begin{array}{l}\text { Tramo VII: } \\
\text { Roncha - } \\
\text { Pucara }\end{array}$ & $\begin{array}{l}\text { IC-Docente I: "Si ayudo } \\
\text { a que vayan más rápido a } \\
\text { sus escuelas, ahora van al } \\
\text { paradero a tomar auto". }\end{array}$ \\
\hline
\end{tabular}

Fuente: Entrevista aplicada a docentes de las diferentes instituciones educativas ubicados del tramo I al tramo VII.

En la figura $2\left(\mathrm{~N}^{\circ}\right.$ de matriculados 2017 vs 2018) y en la tabla 1 (Impacto de la construcción del Corredor Vial Cañete en el servicio de educación) se evidencia los impactos generados por el proyecto en el servicio educación, teniendo como eje principal el acceso a la educación en las diversas instituciones educativas de los diferentes niveles: primario, secundario y universitario. A causa de la construcción del Corredor Vial Cañete, se mejoró el fluido del transporte para que dichos estudiantes y docentes se movilicen fácilmente y obtengan una educación de calidad a la vez permitió que los materiales educativos lleguen con mucha más rapidez contribuyendo a la mejora de los aprendizajes de estos estudiantes y que estos no sean excluidos. Caso contrario sucede en el Tramo II, III, VI, VII y VIII en donde, en el año 2018 el número de los estudiantes matriculados fue menor respecto al 2017, esto debido a que los padres ven como posibilidad una mejor educación fuera del lugar de residencia, consideran que la mejor enseñanza la encuentran en instituciones ubicadas en provincias como Cañete o en la capital. En otro de los casos piensan que los profesores más capacitados enseñan en instituciones privadas y no en las instituciones del estado, por lo que se ven impulsados a mandar a sus hijos fuera de los anexos y en algunos casos son los estudiantes de

educación secundaria los que más se retiran de las instituciones ubicadas en su lugar de residencia, a fin de acceder a estudios superiores universitarios y o técnicos.

Salud

Dentro del sector salud se tomó en cuenta hospitales, centros o puestos de salud que se encuentran a pie de carretera y de tramo en tramo. Así mismo se tomó en cuenta el número de personas atendidas (persona que recibió una atención por primera vez en un periodo determinado, existe la posibilidad que un paciente se atienda en diferentes 
establecimientos de salud sea por referencia, migración, viaje, emergencia, o afiliación en distintas regiones) y el número de atenciones (número de veces o frecuencias de atención del mismo atendido por diversos motivos) las cuales se detallan a continuación.

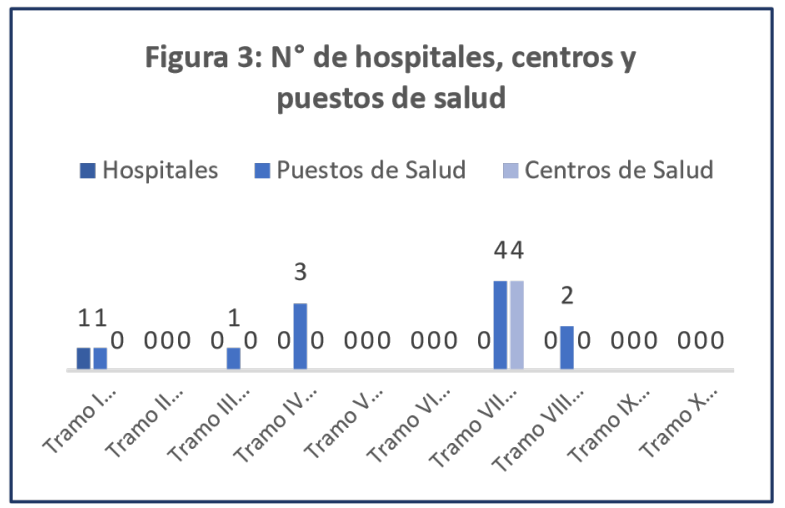

Fuente: Encuesta aplicada a pobladores por cada tramo -2018

Se muestra el número de hospitales, centros y postas de salud en los Tramos del I al X. En el tramo I se encuentra 01 hospital y un puesto de salud, en el Tramo III (1 puesto de salud), en el tramo IV (03 puestos de salud), en el tramo VII ( 4 centros y 4 puestos de salud), en el tramo VIII (2 puestos de salud); mientras que en el Tramo II, V, VI, IX y $\mathrm{X}$, no se encuentra ningún establecimiento de salud.

Figura 4: $\mathrm{N}^{\circ}$ de atenciones 2017 vs 2018

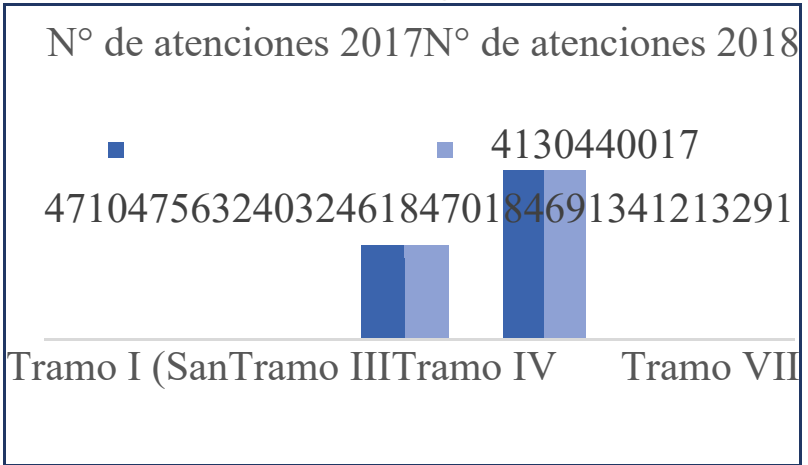

Tramo VIII

Vicente -(Lunahuana(Zuñiga-(Roncha - (Pucará - Imperial )- Zuñiga)Tomás) Pucará) Pazos) 
Fuente: Encuesta aplicada a pobladores por cada tramo -2018

Se muestra el Número de atenciones (frecuencia de atención del mismo paciente). En el tramo I (4.710 4.756) y II (3240- 3246), en el año 2018 se evidencia una progresiva de aumento en el número de atenciones respecto al 2017, debido a que la construcción de la carretera le ha permitido al paciente llegar en menor tiempo al lugar de atención y ya no tiene que verse forzado a caminar.

Así mismo permite a los profesionales en salud llegar hasta los centros poblados antes olvidados con campañas de salud en diferentes temas, con el cual es atendida la necesidad de estas poblaciones vulnerables y en muchos casos la accesibilidad a través de la carretera ha permitido canalizar los casos de enfermedades como las IRAs y las EDAs a otros establecimientos de salud donde cuentan con mejor infraestructura, equipamiento y atención de profesionales.

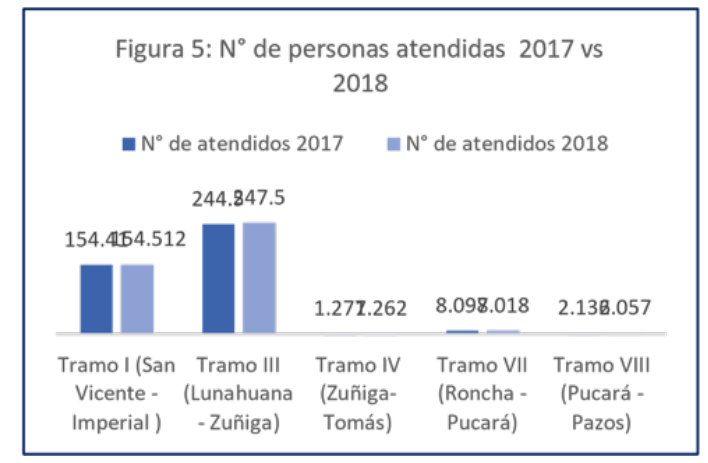

Fuente: Encuesta aplicada a pobladores por cada tramo -2018

Se muestra el número de personas atendidas (persona que recibió una atención por primera vez en un tiempo determinado). En el tramo I, en el año 2018 se observa un crecimiento en el número de atenciones con respecto al 2017 (154.410-154.512) al igual que en el tramo III (244.5 - 247.5), caso contrario sucede en el Tramo IV, VII y VIII en donde se observa que el número de atenciones disminuyo con respecto al año 2018, esto debido a que otros pacientes decidieron continuar su tratamiento en otros centros de salud con

mejor infraestructura y mejores equipos, permitiendo su traslado a estos gracias a la construcción del corredor vial.

Asimismo al preguntar a los doctores y algunos pacientes (informantes claves) acerca de si la construcción del Corredor Vial Cañete, mejoro el servicio de salud de su zona, respondieron:

Tabla 2: Impacto de la construcción del Corredor Vial Cañete en el acceso al servicio de salud 


\begin{tabular}{|c|l|}
\hline & $\begin{array}{l}\text { seguido } \\
\text { controles". }\end{array}$ \\
\hline Tramo VII: & $\begin{array}{l}\text { IC-Paciente I:" Si ayuda } \\
\text { la carretera para poder }\end{array}$ \\
- Pucara & $\begin{array}{l}\text { ir más rápido aL centro } \\
\text { de salud". } \\
\text { IC-Paciente II:" Bueno } \\
\text { para ir a la posta hay } \\
\text { carros o sino a un amigos } \\
\text { con carro propio le pides } \\
\text { ayuda y te lleva". }\end{array}$ \\
\hline
\end{tabular}

Fuente: Entrevista aplicada a doctores y pacientes de los diferentes centros de salud ubicados en los distintos tramos.

En la tabla 2 se evidencia los impactos generados por el proyecto en el acceso al servicio de salud. La Construcción del Corredor Vial Cañete, permitió a los pobladores trasladarse de manera más rápida a los centros de salud; realizar su control médico de manera más frecuente. Asimismo, permite que las ambulancias lleguen más rápido a la zona de emergencia y así trasladar a los heridos en menor tiempo, de igual manera permitió que los medicamentes y profesionales de salud, lleguen a los centros de salud más alejados.

Servicios Básicos

A continuación, se detalla la cantidad de servicios básicos (agua, luz y desagüe) de la población

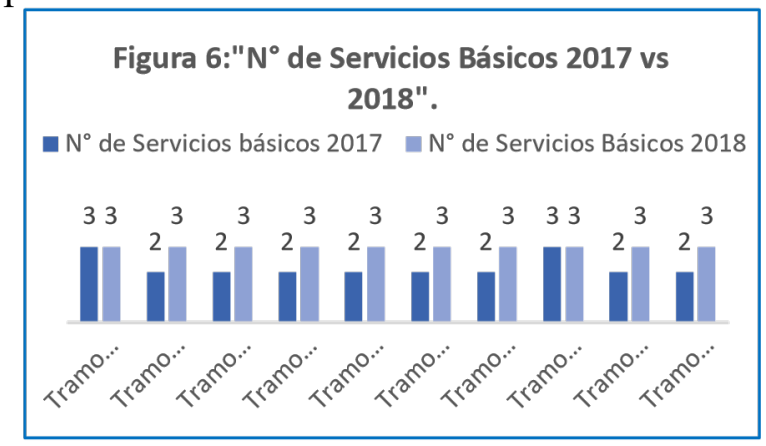

beneficiaria en el año 2017 y 2018.

Fuente: Encuesta aplicada a pobladores de los diferentes tramos - 2018

Se muestra el número de servicios básicos en los tramos del I al X. En el tramo II, III, IV, V, VI, VII, IX y X, se observa un incremento en el número de servicios básicos 
(agua, luz y desagüe) contribuyendo así a la mejora de su calidad de vida. El 95\% de los Distritos y anexos ubicados en los diferentes tramos tienen acceso al servicio de agua, luz y desagüe, las cuales se brindan las 24 horas del día sin ninguna interrupción, en tanto la frecuencia de la prestación y uso de los servicios básicos a cada momento, debido a que los anexos de los tramos en mención son sitios urbano-rurales donde se concentran gran cantidad de personas de los diversos grupos etarios, quienes se dedican a la agricultura , el comercio y en menor parte a la ganadería , así mismo las diversas instituciones que brindan servicio de educación, salud entre otros también cuentan con los servicios básicos siendo estos primordiales para brindar una atención de calidad.

Así mismo al preguntar a los pobladores de los diferentes tramos, sobre si tienen acceso a los servicios básicos, respondieron lo siguiente.

Tabla 3: Acceso a servicios básicos

\begin{tabular}{|c|l|}
\hline \multicolumn{1}{|c|}{ Tramo } & \multicolumn{1}{|c|}{ Informante Clave } \\
\hline $\begin{array}{c}\text { Tramo I: San } \\
\text { Vicente } \\
\text { - Imperial }\end{array}$ & $\begin{array}{l}\text { IC-Poblador:" Tenemos } \\
\text { todos los servicios: Agua, } \\
\text { luz y desagüe”. }\end{array}$ \\
\hline $\begin{array}{l}\text { Tramo II: Nueva } \\
\text { Imperial - } \\
\text { Lunahuana }\end{array}$ & $\begin{array}{l}\text { IC-Poblador:" Si con luz } \\
\text { agua y desagüe". }\end{array}$ \\
\hline $\begin{array}{c}\text { Tramo III: } \\
\text { Lunahuana } \\
\text { - Zúñiga }\end{array}$ & $\begin{array}{l}\text { IC-Poblador: "Solo } \\
\text { carecemos de desagüe”. }\end{array}$ \\
\hline
\end{tabular}

\subsection{Impacto Económico}

En el área de influencia directa, la agricultura constituye la principal actividad económica de las comunidades, así mismo el comercio es otra de las actividades relevantes al cual se dedica la población entrevistada, como es la venta de frutas, verduras, legumbres, atención en restaurantes y hospedajes, vitivinícolas, venta de ganados y sus derivados, servicio de transporte, entre otros, dentro y fuera de la zona. 
Las familias campesinas se dedican también a la crianza de ovinos, equinos, camélidos sudamericanos, cuyes y aves de corral.

Las actividades económicas que realizan los pobladores del tramo I al Tramo X son diversas, considerando que el proyecto abarca tres Regiones del país (Lima, Junín y Huancavelica), cada una de ellas distintas y con características peculiares. Por ello que la presente evaluación socioeconómica se divide en los siguientes tramos:

Tabla 3: Impacto Económico

distritos, mejorando su nivel de ingreso. Asimismo, en el tramo II Y V, la actividad del turismo se posiciono como su principal actividad económica, ya que la construcción de carreteras facilito que los visitantes puedan llegar a los centros turísticos.

Fuente: Entrevista aplicada a pobladores de los diferentes tramos.

En la tabla 3(Impacto económico), se puede evidenciar que la Construcción del Corredor Vial Cañete, produjo un impacto positivo en las actividades económicas de los diferentes pobladores ubicados en los tramos del I al VII. Sobre todo, en la actividad de agricultura y comercio, pues la construcción de carreteras, permite que los comerciantes y agricultores puedan transportar de manera más rápida sus productos a los diferentes

\section{Discusiones}

"Las carreteras constituyen una pieza fundamental e importante en relación al desarrollo económico y social de nuestro país, los cambios generados debido a la globalización han hecho necesarias la creación de sistemas que apunten hacia la mejora de la calidad y rapidez de comunicación por ende demanda mejoras de acceso y construcción de estructuras viales y de infraestructura". En el

Perú el Ministerio de Transportes y Comunicaciones (MTC), "a través de Provias Nacional, realiza el servicio de gestión, mejoramiento y conservación de carreteras".

"En tal sentido, la construcción del Corredor Vial Cañete además de ser considerado un importante corredor para mejorar la accesibilidad a la zona centro del país en vista que agrupa provincias desde Lunahuaná - Dv. Yauyos hasta la división Dv. Pampas y que permite integrar dos regiones del Perú: la costa con la sierra (desde Cañete hasta Huancayo), ha producido impactos sociales y económicos positivos en los pobladores 
de las zonas aledañas al Corredor". El impacto social que ha producido en los pobladores, la construcción del Corredor vial Cañete es: el mayor acceso a la educación, salud (atención médica) y a los servicios básicos. En tal sentido, Lecca C. (2013) en el estudio de rehabilitación de la carretera, señala que la construcción de carreteras genera un positivo índice de desarrollo humano que involucra a la población que es consciente de la ejecución del proyecto y sus beneficios a la sociedad civil.

En lo que refiere al mayor acceso a la educación, uno de los indicadores de la calidad de vida es el acceso a la educación, "que es capaz de contribuir a superar las desigualdades y de reducir la brecha social que actualmente emerge en nuestro país". "La educación bajo el enfoque de inclusión social ha surgido para hacer frente a los altos índices de exclusión, discriminación y a las desigualdades educativas presentes en el sistema educativo del Perú, donde muchas veces la exclusión se debe al escaso acceso a la educación debido a que dichas instituciones educativas están alejadas de la comunidad de origen, por ello se da énfasis en la mejora del capital humano brindando una educación de calidad cerrando las brechas de inaccesibilidad ,los ingresos económicos insuficientes, entre otros aspectos”. Pascal B (1998), en relación a la teoría del impacto de accesibilidad en relación a los efectos estructurales señala: "ya desde los años sesenta se pensaba que construyendo la mayor cantidad de redes de transporte se generaría un mayor desarrollo, llega a la conclusión que existe una relación causa efecto de manera indirecta entre la infraestructura y el desarrollo"

Para hacer frente a ello, "el Estado peruano instrumenta políticas -como en este caso: la construcción o mejora de carreteras, y garantizar que se atienda de modo prioritario a aquella población que tenga mayores carencias y vulnerabilidad permitiendo a las diversas comunidades mejorar el acceso a la educación para aquellos niños(as), adolescentes y jóvenes que se les hace dificultoso llegar a las instituciones educativas". Para Díaz (2017) en los estudios sobre el impacto social y económico de la construcción de carreteras llega a la conclusión que la relación al sector educación ha sido positivamente impactado por la construcción ya que los estudiantes y profesores tiene facilidad para arribar temprano a sus instituciones aportando así a la calidad de vida de los pobladores.

La construcción y el mantenimiento del Corredor Vial Cañete, ha contribuido significativamente porque ha permitido que un gran porcentaje de estudiantes se trasladen a otras instituciones aledañas y lejanas abriendo posibilidades a una mejor 
educación. Que cuenten con mejores condiciones ya sea de infraestructura, enseñanza, docentes, pues para Gonzales y otros (2012) mejorar la estructura vial y la conectividad entre las regiones con gran potencial comercial con el fin de buscar el desarrollo económico impacta de manera positiva por lo tanto la ubicación estratégica requiere de especial atención para los corredores viales.

"Actualmente la población beneficiaria que cuenta con acceso a educación asciende a un total de 24616 de población escolar ubicados en los tramos de estudio" (INEI, 2014), tal como se aprecia en la figura $2\left(\mathrm{~N}^{\circ}\right.$ de matriculados $\left.2017-2018\right)$ en donde se observa un incremento en el número de matriculados a diferencia del año 2017 , a causa que se mejoró el fluido del transporte para que dichos estudiantes y docentes se movilicen fácilmente y obtengan una educación de calidad a la vez permitió que "los materiales educativos lleguen con mucha más rapidez contribuyendo a la mejora de los aprendizajes de estos estudiantes y que estos no sean excluidos" (IC docente). Caso contrario sucede en el Tramo II,III y VI en donde, en el año 2018 el número de los estudiantes matriculados fue menor respecto al 2017, esto debido a que los padres ven como posibilidad una mejor educación fuera del lugar de residencia, consideran que ... "la mejor enseñanza la encuentran en instituciones ubicadas en provincias como Cañete o en la capital" (IC PPFF).

Así mismo manifiestan: “...los profesores más capacitados enseñan en instituciones privadas y no en las instituciones del estado" (IC PPFF), por lo que se ven impulsados a mandar a sus hijos fuera de los anexos y en algunos casos son los estudiantes de educación secundaria los que más se retiran de las instituciones ubicadas en su lugar de residencia, a fin de acceder a estudios superiores universitarios y o técnicos.

Así mismo en relación a la movilidad manifiestan que “...ya no resulta ser un problema para algunos centros poblados del ámbito rural porque ya cuentan con accesibilidad al igual que otros sitios ubicadas en el ámbito urbano..." (IC PPFF)

En lo que refiere al mayor acceso a la salud (atención médica): "los medios de trasporte han sido de gran utilidad para el traslado de los pacientes en situación de urgencia y emergencia que viven en zonas lejanas hacia la ciudad la ambulancia es uno de los tipos de vehículos de los centros hospitalarios"...(IC Medico),. “Asimismo, la mejora de la carretera, ha permitido el mayor acceso a salud, reduciendo tiempo de traslado de un de un lugar a otro, inclusive la elección de atenderse en un centro o puesto de mayor envergadura o nivel de atención según el paciente o usuario lo requiera”, tal como se 
aprecia en la figura $4\left(\mathrm{~N}^{\circ}\right.$ de personas atendidos) y $5\left(\mathrm{~N}^{\circ}\right.$ de atenciones) en donde se evidencia una progresiva de aumento en el número de atenciones y de personas atendidas, debido a que la construcción de la carretera le ha permitido al paciente llegar en menor tiempo al lugar de atención y ya no tiene que verse forzado a caminar. Handy S y Niemeier D (1997) en relación a la teoría de accesibilidad territorial e impacto de accesibilidad "han realizado un análisis de los múltiples indicadores de accesibilidad y su evolución en el tiempo, y como estos dependen de tres factores: el costo de desplazamiento (dinero y tiempo), grupo de destinos accesibles y modos de transporte”. Asimismo, "estos autores agrupan los indicadores en tres categorías: oportunidades acumulativas (miden las actividades factibles en cada tramo de distancia), indicadores gravitatorios (miden las oportunidades en base al tiempo o costo del desplazamiento) y por último indicadores de utilidad (miden la accesibilidad en relación a las preferencias)".

En relación al aporte de los profesionales en salud les llegar hasta los centros poblados antes olvidados con campañas de salud en diferentes temas, con el cual es atendida la necesidad de estas poblaciones vulnerables y en muchos casos la accesibilidad a través de la carretera ha permitido canalizar los casos de enfermedades como las IRAs y las EDAs a otros establecimientos de salud donde cuentan con mejor infraestructura, equipamiento y atención de profesionales. Neff, M. (1989) "la Teoría indica que el desarrollo se refiere a las personas y no a lo material, y que el mejor proceso de desarrollo es aquel que permita elevar y mejorar más la Calidad de vida de las personas, y esto depende de las oportunidades que tengan las personas para satisfacer sus necesidades básicas".

En relación al mayor acceso a los servicios básicos, la construcción del Corredor Vial Cañete, permitió sobretodo que la población de la zona rural, cuente con el servicio básico de agua, luz y desagüe, contribuyendo así a la mejora de su calidad de vida, reduciendo así las disparidades sociales, tal como se aprecia en la figura 5 ( $\mathrm{N}^{\circ}$ de servicios básicos) en donde se evidencia que el 95\% de los Distritos y anexos ubicados en los diferentes tramos tienen acceso al servicio de agua, luz y desagüe, las cuales se brindan las 24 horas del día sin ninguna interrupción, “en tanto la frecuencia de la prestación y uso de los servicios básicos a cada momento, debido a que los anexos de los tramos en mención son sitios urbano-rurales donde se concentran gran cantidad de personas de los diversos grupos etarios, quienes se dedican a la agricultura, el comercio 
y en menor parte a la ganadería", así mismo las diversas instituciones que brindan servicio de educación, salud entre otros también cuentan con los servicios básicos siendo estos primordiales para brindar una atención de calidad. En tal sentido, Cahuaya E y Sánchez A (2016) en un estudio realizado sobre análisis de impactos de la construcción de la carretera en un tramo del Corredor Vial Cañete señalan que existe un aumento considerable en el comercio, turismo, mejoras en el acceso a la educación y salud, como también la creación de nuevas actividades económicas gracias a las oportunidades que trae consigo la construcción de la carretera

Asimismo, "es preciso mencionar que, con la construcción y ampliación de redes de alcantarillado y desagüe, se redujo significativamente la contaminación ambiental, ya que se desechaba las aguas servidas en el rio más cercano generando que se proliferen diferentes enfermedades repercutiendo en las poblaciones vulnerables del mismo modo generando una contaminación al medio ambiente", citando así a Díaz (2017), quien en su investigación sobre el impacto social y económico de la construcción de la carretera concluye que "sector educación ha sido positivamente impactado por la construcción de esta carretera, ya que los estudiantes y profesores tienen facilidad para llegar temprano a las instituciones, del mismo modo se aprecia un efecto positivo en salud ya que existen menos enfermedades, también existe un efecto positivo en el sector agrícola y en el transporte, mejorando así la calidad de vida de los pobladores de ambos distritos".

"En el área de influencia directa del corredor Vial Cañete, la agricultura constituye la principal actividad económica de las comunidades, así mismo el comercio que marcha en interrelación con el turismo, entre otra de las actividades relevantes al cual se dedica la población entrevistada". La construcción del Corredor Vial Cañete ha brindado a la población facilidades para trasladar sus productos a los distritos aledaños como son: Cañete, Imperial y Nueva Imperial, mejorado así sus ingresos económicos mínimos percibidos, el comercio resulto favorable en el año 2018 a diferencia del 2017, debido a la afluencia de transporte en la zona, ... "que permite que los pobladores puedan trasladar y expender sus productos con mayor fluidez y rapidez a otros distritos" (IC Poblador). Asimismo sus principales actividades económicas son la agricultura y el turismo, siendo este último, la fuente económica más importante, gracias a la afluencia de trasporte los visitantes concurren a las zonas ubicadas dentro de los tramos, de forma que los pobladores expenden sus productos y /u ofrecen sus servicios como: tiendas vitivinícolas a mayor y menor escala, venta de miel, fruterías, agencia de turismo, 
alojamiento, atención en restaurants, áreas de diversión, transporte permitiendo una mejor estabilidad económica y la mejora en sus ingresos económicos.

Asimismo, la construcción del corredor vial ha permitido el acceso de los pobladores a nuevos centros de empleo, debido a que el mantenimiento de la carretera les ha brindado facilidades de traslado a sus centros de trabajo, tal como señala (IC Poblador) de San Vicente de Cañete: "Viajo a diario por motivos laborales... A los diversos colegios de San Vicente e Imperial", de igual manera en el Tramo III existen pobladores que laboran de forma dependiente, en lugares contiguas de la zona, en trabajos como: construcción, atención en hospedaje, atención en boticas, se desplazan caminando o hacen uso de trasporte de servicio público que les permite llegar en menor tiempo, lo mismo que ocurre en los demás tramos. Con lo cual se puede afirmar que el mantenimiento y/o conservación de la carretera ha beneficiado a los pobladores económicamente de forma indirecto o directa, puesto que trasladan y expenden sus productos en los diferentes los mercados de otros distritos donde antes no lo hacían, ha generado oportunidades de venta para los comerciantes dentro de la zona, los pobladores se trasladan a sus centros de trabajo en menor tiempo y hay un incremento considerable de servicio de trasporte. En relación a los descrito Lecca C. (2013) señala que en la dimensión de economía se presentan oportunidades de crecimiento para las pequeñas empresas y comercializadores, facilitando el acceso a la transpirabilidad, del mismo también genera un desarrollo local ya que se descentralizan las gestiones y existe un desarrollo institucional, llegando a la conclusión que dado el impacto positivo que ha tenido es recomendable impulsar el proyecto,

Así mismo otro de los impactos económicos de la construcción del Corredor Vial Cañete, "es el desarrollo de nuevas actividades económicas, siendo uno de ellas el turismo, que marcha en interrelación con el comercio, existiendo entre ellos restaurantes, hospitales, agencias de turismo, bodegas de abarrotes, empresas vitivinícolas, panaderías; por la mejora de las vías de acceso”. El turismo se ha convertido en la fuente económica más importante en el Tramo II, donde se evidencia que gracias a la afluencia de transporte los visitantes concurren a las zonas ubicadas dentro del tramo, de forma que los pobladores expenden sus productos y /u ofrecen sus servicios como: tiendas vitivinícolas a mayor y menor escala, venta de miel, fruterías, agencia de turismo, alojamiento, atención en restaurants, áreas de diversión, transporte permitiendo una mejor estabilidad económica. Por su parte, Montagut J y Patiño C 
(2015) quienes en su trabajo de investigación sobre "el impacto de los proyectos de infraestructura vial en los aspectos socioeconómicos y los cambios en los valores del terreno, manifiestan que, gracias a los proyectos de infraestructura, las actividades comerciales se ven en asenso ya que existen construcciones y aperturas de comercio y turismo" (mercados comerciales, hoteles, etc.) Resulta preciso mencionar, que la construcción y el mantenimiento del Corredor Vial Cañete, contribuye en el logro de los Objetivos de Desarrollo Sostenible (ODS 3,4,9,11 y 17)

Finalmente, a modo de conclusión que la construcción del corredor vial Cañete impactó positivamente en los cambios de calidad de vida de la población, produciendo impactos sociales y económicos de manera individual y colectiva que impulsan un desarrollo sustentable de las áreas de influencia de la carretera. Dentro de los impactos sociales se describe el mayor acceso a la educación reducción de tiempos de transporte para acceder a la educación (evolución en el tiempo: costo de desplazamiento, grupo de destinos accesibles y modos de transportes), mayor acceso del derecho de la educación de calidad (elección entre el lugar de residencia y la ubicación de su institución educativa - nivel de accesibilidad) ; y atención en médica debido a la atención inmediata en relación al traslado de los pacientes (uso de ambulancia), acceso a la salud con mayor fluidez permitiendo reducción de tiempos en atención de emergencias y urgencias, disminución de enfermedades (IRAs y EDAs). Y dentro del impacto económico mayor acceso a mercados (cultivos y productos locales), el acceso a nuevos centros de empleo, desarrollo de nuevas actividades económicas y el fortalecimiento de la economía, logrando impactar positivamente en la reducción de pobreza

Agradecimientos.

Expresamos nuestros agradecimientos a las personas que han contribuido con la investigación: Ingeniero Civil Luis Pineda Mori, Licenciado en Antropología

David Vila Pihue, Ingeniero José Pérez Beraún, Licenciada en Trabajo Social Karol Huilca De la Peña, y estudiantes de la Facultad de Trabajo Social de la Universidad Nacional del Centro del Perú Luz Clara Cañari Oscanoa, Mari Cruz Yesenia Navarro Escabel. Como también a los pobladores, autoridades, docentes y personal de salud entrevistados. 
Conflictos de Interés.

No existen conflictos de interés

Referencias Bibliográficas:

Cahuaya E., Chávez A. (2016). Tesis: “Análisis del impacto de la construcción en una carretera en una zona rural: El caso del

Distrito de Zúñiga en Cañete”. Pontificia Universidad Católica del Perú, Lima.

Consorcio Instituto Cuanto-Macroconsult S.A. (2014). Elaboración de la Evaluación de Impacto y la Ampliación de la Línea de Base del Programa de Transporte Rural Descentralizado (PTRD).

Gonzales y otros (2012) "Impacto socioeconómico causado por los problemas de movilidad entre el área metropolitana de Bucaramanga y

Barrancabermeja", Colombia

Handy, S. y Niemeier, D. (1997).

Measuring

Accessibility: An Exploration of

Issues and

Alternatives. Environment and Planning A 29

INEI (2013). “Evolución de la pobreza monetaria 20072012- Informe Técnico".

INEI (2014). Informe Técnico de la Evaluación de Pobreza Monetaria 2009-2013.

Consulta: Lunes 9 de junio, 2014. Hora 10:15 am. Sitio web:

www.inei.gob.pe/media/cifras_de_po breza/i nformetecnico.pdf

INEI 2012, Perú: Estimaciones y Proyecciones de Población Total por Sexo de las Principales Ciudades 2000-2015. Boletín Especial N²3. Consulta: 
Miércoles 2 de julio, 2014. Hora

9:15 am. Sitio web: proyectos.inei.gob.pe/web/biblioineip ub/ba ncopub/Est/Lib1020/Libro.pdf

Lecca C (2013) “La rehabilitación de la carretera, tramo: Puente Pallar - EL

molino; y su impacto social y económico de la provincia de Sánchez Carrión”, Arequipa

Meza S. (2016) La vivienda social en el Perú. Evaluación de las políticas

y programas sobre vivienda de interés social. Caso de estudio: Programa "Techo Propio".

Ministerio de Economía y Finanzas (MEF) del Perú Ministerio de Economía y

Finanzas (2007). Población sin acceso a servicios básicos. Sitio web: www.mef.gob.pe/index.php?option $=\mathrm{c}$ om co ntent\&view $=$ article $\& \mathrm{id}=266 \&$ Itemid $=100$

Ministerio de Transportes y Comunicaciones (2010). Estudio de mantenimiento periódico de la carretera panamericana norte.

Ministerio de Transportes y Comunicaciones (2013). Manual de

Carreteras: Especificaciones técnicas generales para construcción EG2013.

Ministerio de Transportes y Comunicaciones (2013). Manual de Carreteras:

Montagut J y Patiño C (2015) "El impacto de los proyectos de infraestructura vial en los aspectos socioeconómicos y los cambios en los valores del terreno en una zona en del Departamento del Cesar", Colombia.

Neff, M. (1989) Teoría de las necesidades humanas para el desarrollo

Pascal, B. (1998). Analyser les mobilités et le rayonnement des villes. Les Cahiers Scientifiques du Transport, $\mathrm{N}^{\circ} 33$,

1998. Recuperado Diciembre 2004. Sitio web: www.afitl.com/CST/Contenu\%20des \%20pr\% C3\%A9c\%C3\%A9dents\%20num\%C3 \%A9ros/N33/BERION33.PDDíaz

Política General de Gobierno y el Plan Estratégico de Desarrollo Nacional” (PESEM: Sector de transporte y comunicaciones 2018-2023).

Puyo R (2012) Del campo a la ciudad. El proceso de urbanización en el mundo 